\title{
The Ebers-Moll model for magnetic bipolar transistors
}

\author{
Jaroslav Fabian ${ }^{\text {a) }}$ \\ Institute of Physics, Karl-Franzens University, Universitätsplatz, 5, 8010 Graz, Austria \\ Igor Žutićs) \\ Center for Computational Materials Science, Naval Research Laboratory, Washington, D.C. 20375 \\ and Condensed Matter Theory Center, Department of Physics, University of Maryland at College Park, \\ College Park, Maryland 20742-4111
}

(Received 20 September 2004; accepted 15 February 2005; published online 23 March 2005)

\begin{abstract}
The equivalent electrical circuit of the Ebers-Moll-type is introduced for magnetic bipolar transistors. In addition to conventional diodes and current sources, the new circuit comprises two novel elements due to spin-charge coupling. A classification scheme of the operating modes of magnetic bipolar transistors in the low bias regime is presented. (C) 2005 American Institute of Physics. [DOI: 10.1063/1.1886251]
\end{abstract}

Semiconductor spintronics ${ }^{1}$ offers novel functionalities by combining electronics for signal processing and magnetism for both nonvolatility and additional electronic control. At the present stage, with the fundamentals of spin injection, ${ }^{2-5}$ spin relaxation, ${ }^{6-8}$ as well as semiconductor magnetism ${ }^{9,10}$ established, there is a need for new ideas demonstrating practical use of the fundamental spin physics. It was shown in Refs. 11-13 that magnetic bipolar transistors (MBT), which can employ both ferromagnetic and paramagnetic semiconductors (with large $g$-factor), ${ }^{1}$ can significantly extend functionalities of conventional bipolar junction transistors (BJT) (Ref. 14) by exploiting spin-charge coupling of the Silsbee-Johnson-type. ${ }^{15,16}$ Current amplification in MBT's, for example, can be modulated by magnetic field during the device operation, giving rise to the phenomena of (giant) magnetoamplification. ${ }^{13}$

In this paper we generalize the widely used Ebers-Moll equivalent circuit of BJT (Ref. 17) (reprinted in Ref. 18) to MBT. Two novel electronic elements are added to the original circuit-spin diodes and spin current sources-to describe spin-charge coupling. Our goal is to provide a simple computational scheme for MBT's as well as to show an example how a novel spintronics device can be described by (and integrated with) a more conventional electronic circuitry.

MBT's comprise two magnetic $p-n$ junctions ${ }^{19,20}$ in series. In the scheme of Fig. 1 we show an npn MBT with a magnetic base. Magnetic here means that there is an equilibrium spin splitting $2 q \zeta_{b}$ of the conduction band (valence band would also work), giving rise to an equilibrium spin polarization $P_{0 b}=\tanh \left(q \zeta_{b} / k_{B} T\right)$ in the base ( $T$ is temperature and $q$ is the proton charge). Hole spins are assumed unpolarized. Either a ferromagnetic semiconductor (with conduction or impurity band free carriers) or a diluted magnetic semiconductor with a large $g$-factor in a magnetic field will work. Both the magnitude and the sign of $P_{0 b}$ can be controlled by an external magnetic field (this is what we call magnetic control). In addition to the equilibrium spin, there can be a nonequilibrium (excess) spin injected by external means

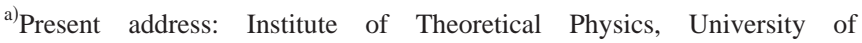
Regensburg, 93040 Regensburg, Germany; electronic mail: jaroslav.fabian@physik.uni-regensburg.de

${ }^{b}$ Electronic mail: zutic@dave.nrl.navy.mil
}

(providing spin control) into the emitter and collector. The corresponding spin polarizations are $\delta P_{e}$ and $\delta P_{c}$. If the bias on the base-emitter (be) and base-collector (bc) junction is $V_{\text {be }}$ and $V_{\text {bc }}$ respectively, then the excess, $\delta n=n-n_{0}$, where $n_{0}$ is the equilibrium number, electron densities in the base, close to the be and bc junctions, are ${ }^{13}$

$$
\begin{aligned}
& \delta n_{\mathrm{be}}=n_{0 b}\left(\zeta_{b}\right)\left[e^{q V_{\mathrm{be}} / k_{B} T}\left(1+\delta P_{e} P_{0 b}\right)-1\right], \\
& \delta n_{\mathrm{bc}}=n_{0 b}\left(\zeta_{b}\right)\left[e^{q V_{\mathrm{bc}} / k_{B} T}\left(1+\delta P_{c} P_{0 b}\right)-1\right] .
\end{aligned}
$$

The influence of the equilibrium spin is felt both by the equilibrium number of electrons in the base, $n_{0 b}\left(\zeta_{b}\right)$ $=n_{0 b}(0) \cosh \left(q \zeta_{b} / k_{B} T\right)$, as well as by the spin-charge coupling factor $1+\delta P P_{0 b}$. The nonequilibrium spin plays a role only in the latter. The unpolarized hole excess densities in the emitter and collector, close to the depletion layer with the base, are given by the standard formulas

$$
\delta p_{e}=p_{0 e}\left(e^{q V_{\mathrm{be}} / k_{B} T}-1\right),
$$
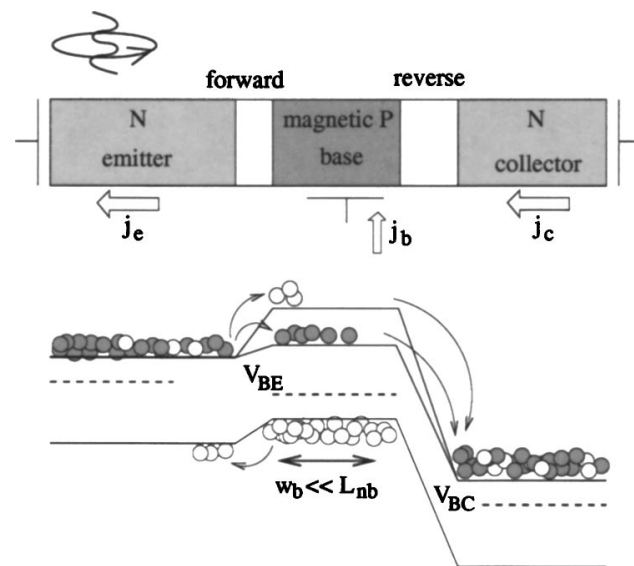

FIG. 1. Scheme of a npn magnetic bipolar transistor in the forward active mode. The base has an equilibrium electron spin polarization $P_{0 b}$, illustrated by the spin-split conduction band. Spin up (down) electrons are pictured as dark (light) filled circles. Holes are unpolarized. The emitter has a source of spin polarization, here shown as a circularly polarized light, giving rise to a nonequilibrium spin polarization $\delta P_{e}$. The direction of the currents is indicated. 


$$
\delta p_{c}=p_{0 c}\left(e^{q V_{\mathrm{bc}} / k_{B} T}-1\right),
$$

where $p_{0 e}$ and $p_{0 c}$ is the equilibrium number of holes in the emitter and collector.

Electrical currents in MBT's can be expressed, as in BJT's through the excess densities $\delta n_{\text {be }}$ and $\delta n_{\text {bc }}$ of the minority carriers $^{13}$

$$
\begin{aligned}
& j_{e}=j_{g b}^{n}\left[\frac{\delta n_{\mathrm{be}}}{n_{0 b}}-\frac{1}{\cosh \left(w_{b} / L_{n b}\right)} \frac{\delta n_{\mathrm{bc}}}{n_{0 b}}\right]+j_{g e}^{p} \frac{\delta p_{e b}}{p_{0 e}}, \\
& j_{c}=j_{g b}^{n}\left[-\frac{d n_{\mathrm{bc}}}{n_{0 b}}+\frac{1}{\cosh \left(w_{b} / L_{n b}\right)} \frac{\delta n_{\mathrm{be}}}{n_{0 b}}\right]-j_{g c}^{p} \frac{\delta p_{c b}}{p_{0 c}} .
\end{aligned}
$$

The base current is $j_{b}=j_{e}-j_{c}$ and the electron generation current in the base is

$$
j_{g b}^{n}=\frac{q D_{n b}}{L_{n b}} n_{0 b} \operatorname{coth}\left(\frac{w_{b}}{L_{n b}}\right) .
$$

Here $D_{n b}$ stands for the electron diffusion coefficient in the base whose effective width is $w_{b}$ and $L_{n b}$ is the electron diffusion length in the base (see Fig. 1). The hole generation currents in the emitter, $j_{g e}^{p}$, and collector, $j_{g c}^{p}$, are given similarly to Eq. (7) with $n$ replaced by $p$ and $e$ replaced by either $e$ or $c$.

The Ebers-Moll model ${ }^{17}$ is an equivalent circuit to a BJT. We will now introduce this standard model and generalize it to the case of MBT's. Denote by $j_{s e}$ and $j_{s c}$ the emitter and collector saturation currents (note that $s$ stands for saturation, not spin):

$$
\begin{aligned}
& j_{s e}=j_{g b}^{n}+j_{g e}^{p}, \\
& j_{s c}=j_{g b}^{n}+j_{g c}^{p} .
\end{aligned}
$$

The emitter saturation current is the emitter current that flows if $V_{\mathrm{be}}<0, V_{\mathrm{bc}}=0$, and only equilibrium spin present $\left[j_{e}=-j_{s e}\right.$, see Eq. (5)]. Similarly for the collector saturation current. Denote next the forward and reverse currents (terminology from the forward active mode) as

$$
\begin{aligned}
& j_{f}=j_{s e}\left(e^{q V_{\mathrm{be}} / k_{B} T}-1\right), \\
& j_{r}=j_{s c}\left(e^{q V_{\mathrm{bc}} / k_{B} T}-1\right) .
\end{aligned}
$$

Finally, we introduce the spin-charge forward and reverse currents

$$
\begin{aligned}
& j_{m f}=j_{g b}^{n} \delta P_{e} P_{0 b} e^{q V_{\mathrm{be}} / k_{B} T}, \\
& j_{m r}=j_{g b}^{n} \delta P_{c} P_{0 b} e^{q V_{\mathrm{bc}} / k_{B} T} .
\end{aligned}
$$

Here subscript $m$ stands for magnetic to stress that the current, that is due to spin-charge coupling across the depletion regions, appears only in magnetic transistors. These currents flow due to the presence of nonequilibrium spin polarization and are finite even at zero bias (spin-voltaic effect ${ }^{19}$ ).

The generalized Ebers-Moll model directly derives from Eqs. (5) and (6), and reads

$$
\begin{aligned}
& j_{e}=j_{f}-\alpha_{r} j_{r}+j_{m f}-\alpha_{t} j_{m r}, \\
& j_{c}=\alpha_{f} j_{f}-j_{r}+\alpha_{t} j_{m f}-j_{m r} .
\end{aligned}
$$

Here $\alpha_{f}$ has the meaning of the transport factor in the forward active mode, while $\alpha_{r}$ is the transport factor in the reverse active mode in the absence of spin-charge coupling,

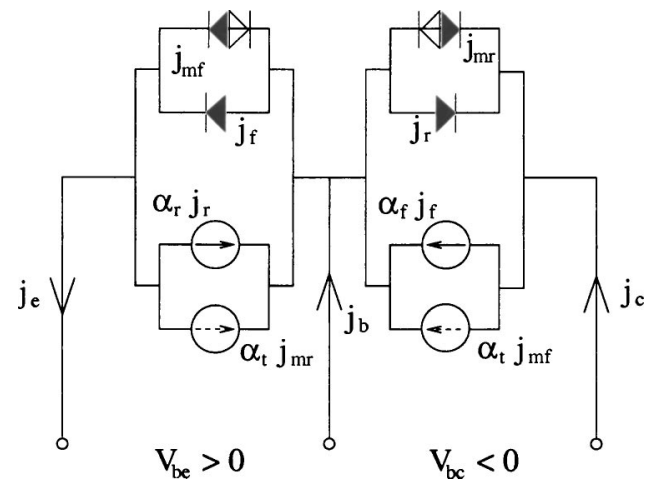

FIG. 2. The Ebers-Moll equivalent circuit of a MBT. The voltage sources are arranged for the forward active mode. The left (right) circuit is the emitter (collector). The emitter circuit has a diode for the forward current, and a current source which depends on the bias in the collector circuit. In addition, there are two new elements. A spin diode whose direction can be flipped: its filled triangle points to the forward direction when $\delta P_{e} P_{0 b}>0$, otherwise the current direction changes. A new spin current source (dashed arrow), pointing in the direction of the current if $\delta P_{e} P_{0 b}>0$. The direction of the current can also be flipped. Similar notation applies for the right (collector) circuit.

as can be seen directly from Eqs. (14) and (15). The transport factor $\alpha_{t}$ is $\alpha_{t}=1 / \cosh \left(w_{b} / L_{n b}\right)$. The conventional EbersMoll model is recovered by putting $j_{m f}=j_{m r}=0$. As in the conventional model, the following equality holds:

$$
\alpha_{f} j_{s e}=\alpha_{r} j_{s c} .
$$

This can be verified by requiring that

$$
j_{e}\left(V_{\mathrm{be}}=0, V_{\mathrm{bc}}=V\right)=-j_{c}\left(V_{\mathrm{be}}=V, V_{\mathrm{bc}}=0\right),
$$

for $\delta P_{e}=\delta P_{c}=0$. In our ideal case it is straightforward to show that

$$
\alpha_{f} j_{s e}=\alpha_{r} j_{s c}=\alpha_{t} j_{g b}^{n} .
$$

The equivalent circuit to Eqs. (14) and (15) is shown in Fig. 2. The current flow is the same as in Fig. 1. Let us discuss the emitter circuit. It consists of four elements: (i) a conventional diode with the directional current $j_{f}$ that depends on $V_{\text {be }}$, (ii) a conventional current source giving current $\alpha_{r} j_{r}$ that depends on $V_{\mathrm{bc}}$ and on the transport factor $\alpha_{r}$ measuring the amount of current injected into the emitter from the collector, (iii) a spin diode with the forward current $j_{m f}$, and finally, (iv) a spin current source $\alpha_{t} j_{m r}$. The first two elements appear already in BJT's. The spin diode (iii), which appears due to spin-charge coupling, works similar to a diode in the sense that its current is rectified with $j_{m f}$ $\sim \exp \left(q V_{\mathrm{be}} / k_{B} T\right)$. The crucial difference from conventional diodes is that the direction of the current flow can be changed by changing the sign of $\delta P_{e} P_{0 b}$, see Eq. (12). The symbol for the spin diode reflects this fact. The filled triangle shows the direction when $\delta P_{e} P_{0 b}$ is positive. The new functionality of MBT's then lies in the ability to switch or modify the spin diode during its operation. There is, in addition, the spin current source (iv) that is due to the electron current from spin-charge coupling. The current, injected into the base from the collector, diffuses towards the emitter through the base (this is why the transport factor $\alpha_{t}$ appears). The element is a current source because it does not depend on the voltage drop (here $V_{\mathrm{be}}$ ) across it. It is, however, a controlled current source, similar to (ii), because it can be controlled by $V_{\mathrm{bc}}$. Because it arises from spin-charge coupling, the magni-
to AlP license or copyright, see http://apl.aip.org/apl/copyright.jsp 
TABLE I. Operational modes of BJT's and MBT's. Forward (F) and reverse (R) bias means positive and negative voltage, respectively. Symbols MA and GMA stand for magnetoamplification and giant magnetoamplification, while $\mathrm{ON}$ and $\mathrm{OFF}$ are modes of small and large resistance, respectively; SPSW stands for spin switch.

\begin{tabular}{ccccc}
\hline \hline Mode & $V_{\mathrm{be}}$ & $V_{\mathrm{bc}}$ & BJT & MBT \\
\hline Forward active & $\mathrm{F}$ & $\mathrm{R}$ & amplification & MA, GMA \\
Reverse active & $\mathrm{R}$ & $\mathrm{F}$ & amplification & MA, GMA \\
Saturation & $\mathrm{F}$ & $\mathrm{F}$ & ON & ON, GMA, SPSW \\
Cutoff & $\mathrm{R}$ & $\mathrm{R}$ & OFF & OFF \\
Spin-voltaic & 0 & 0 & OFF & SPSW \\
\hline \hline
\end{tabular}

tude and direction of (iv) can be controlled by spin and magnetic field, adding to the functionality of (iii). Similar description applies to the collector circuit.

For completeness we summarize in Table I the operating modes (for a textbook discussion see, for example, Ref. 21) of both BJT's and MBT's, described by the Ebers-Moll model. Conventional transistors have four modes, with amplification only in the forward and reverse active modes (due to design only the forward active mode has significant current gain). The saturation and cutoff modes are used in logic circuits for ON and OFF states, respectively. MBT's have a much richer structure. In the active modes both magnetoamplification $^{11,13,22}$ due to the dependence of the saturation currents on the equilibrium spin polarization and giant magnetoamplification ${ }^{13}$ due to spin-charge coupling appear. In contrast to conventional transistors, MBT's provide current gain even in the saturation mode, due to spin-charge coupling. Furthermore, the transistor can act as a spin switch, switching the current direction by flipping the $\operatorname{spin}^{23}$ In the cutoff mode MBT's are OFF and spin effects are inhibited [see Eqs. (12) and (13)]. Finally, a qualitatively new mode, spin-voltaic, appears, due to spin-charge coupling. In this mode, with no applied biases, the currents that flow are due only to the presence of nonequilibrium spin (which provide spin emf) and MBT's act as spin switches.
In summary, we have generalized the Ebers-Moll model to include spin-charge coupling and cover magnetic bipolar transistors. We have classified different operating modes of the transistors. In most modes MBT's offer new functionalities such as spin switches or magnetoamplifiers, which may have potential for signal processing, logic circuits and nonvolatile memories.

This work was supported by the U.S. ONR, NSF, and DARPA. I. Ž. acknowledges financial support from the National Research Council.

${ }^{1}$ I. Žutić, J. Fabian, and S. Das Sarma, Rev. Mod. Phys. 76, 323 (2004).

${ }^{2}$ R. Fiederling, M. Kleim, G. Reuscher, W. Ossau, G. Schmidt, A. Waag, and L. W. Molenkamp, Nature (London) 402, 787 (1999).

${ }^{3}$ D. K. Young, E. Johnston-Halperin, D. D. Awschalom, Y. Ohno, and H. Ohno, Appl. Phys. Lett. 80, 1598 (2002).

${ }^{4}$ B. T. Jonker, Y. D. Park, B. R. Bennett, H. D. Cheong, G. Kioseoglou, and A. Petrou, Phys. Rev. B 62, 8180 (2000).

${ }^{5}$ X. Jiang, R. Wang, S. van Dijken, R. Shelby, R. Macfarlane, G. S. Solomon, J. Harris, and S. S. P. Parkin, Phys. Rev. Lett. 90, 256603 (2003).

${ }^{6}$ Optical Orientation, edited by F. Meier and B. P. Zakharchenya (NorthHolland, New York, 1984).

${ }^{7}$ J. M. Kikkawa and D. D. Awschalom, Phys. Rev. Lett. 80, 4313 (1998).

${ }^{8}$ R. I. Dzhioev, V. L. Korenev, B. P. Zakharchenya, D. Gammon, A. S. Bracker, J. G. Tischler, and D. S. Katzer, Phys. Rev. B 66, 153409 (2002).

${ }^{9}$ T. Dietl, Semicond. Sci. Technol. 17, 377 (2002).

${ }^{10}$ H. Ohno, Science 281, 951 (1998).

${ }^{11}$ J. Fabian, I. Žutić, and S. Das Sarma, cond-mat/0211639 (2002).

${ }^{12}$ J. Fabian, I. Žutić, and S. Das Sarma, Appl. Phys. Lett. 84, 85 (2004).

${ }^{13}$ J. Fabian and I. Žutić, Phys. Rev. B 69, 115314 (2004).

${ }^{14}$ W. Shockley, M. Sparks, and G. K. Teal, Phys. Rev. 83, 151 (1951).

${ }^{15}$ R. H. Silsbee, Bull. Magn. Reson. 2, 284 (1980).

${ }^{16}$ M. Johnson and R. H. Silsbee, Phys. Rev. Lett. 55, 1790 (1985).

${ }^{17}$ J. J. Ebers and J. L. Moll, Proc. IRE 42, 1761 (1954).

${ }^{18}$ J. J. Ebers and J. L. Moll, in Semiconductor Devices: Pioneering Papers, edited by S. M. Sze (World Scientific, Amsterdam, 1991), pp. 276-287.

${ }^{19}$ I. Žutić, J. Fabian, and S. Das Sarma, Phys. Rev. Lett. 88, 066603 (2002).

${ }^{20}$ J. Fabian, I. Žutić, and S. Das Sarma, Phys. Rev. B 66, 165301 (2002).

${ }^{21}$ S. Dimitrijev, Understanding Semiconductor Devices (Oxford University Press, New York, 2000).

${ }^{22}$ N. Lebedeva and P. Kuivalainen, J. Appl. Phys. 93, 9845 (2003).

${ }^{23}$ J. Fabian and I. Žutić, Acta Phys. Pol. A 106, 109 (2004). 